\title{
European Respiratory Society International Congress programme highlights on occupation and epidemiology
}

\author{
Carl Reynolds, Paul Cullinan \\ Faculty of Medicine, National Heart \& Lung Institute, Royal Brompton Campus, Imperial College London, South Kensington Campus, London, UK \\ Correspondence to: Carl Reynolds. Faculty of Medicine, National Heart \& Lung Institute, Royal Brompton Campus, Imperial College London, South \\ Kensington Campus, London, UK. Email: carl.reynolds@imperial.ac.uk; Paul Cullinan. Faculty of Medicine, National Heart \& Lung Institute, \\ Royal Brompton Campus, Imperial College London, South Kensington Campus, London, UK. Email: p.cullinan@imperial.ac.uk.
}

Submitted Aug 10, 2017. Accepted for publication Sep 09, 2017.

doi: $10.21037 /$ jtd.2017.11.45

View this article at: http://dx.doi.org/10.21037/jtd.2017.11.45

\section{Genes in asthma}

Asthma is a common chronic childhood respiratory disease long-known to be both strongly heritable and to have important environmental determinants. In recent years genome-wide association studies (GWAS) have emerged as an indispensable tool to help understand the complex geneenvironment relationships that determine asthma susceptibility and phenotype, including response to treatment.

Three studies presented in abstract at this year's meeting of the ERS illustrate the utility of GWAS in understanding asthma susceptibility and phenotype. Researchers from Bristol in the United Kingdom identified a potential set of exclusive markers for the subphenotype of early onset wheeze that remits by age 11 (1). Authors from Nottingham in the United Kingdom presented findings from the largest casecontrol GWAS study to date of moderate-severe asthma, 5,135 individuals of European ancestry with British Thoracic Society defined asthma and 25,675 controls free from lung disease, which included the identification of multiple novel susceptibility loci (2). Finally, researchers from Brisbane, Australia presented data from a GWAS of 360,838 patients to identify genetic risk factors underpinning the well-recognised clinical associations of asthma, hay fever, and atopic eczema.

Epigenetic mechanisms in asthma are now widely recognised by researchers to mediate gene-environment relationships, playing an important role in asthma pathogenesis, and potentially helping to explain observed phenotypic variations; air pollution for example has been associated with DNA methylation in some studies. Authors from Grenoble, France, presented interesting and novel work identifying $\mathrm{CpG}$ methylation sites associated with reduced $\mathrm{FEV}_{1}$ in persons with asthma using MethylC-capture sequencing (a high-throughput method measuring DNA methylation level) in cohort of 357 adults with the condition (3).

GWAS studies in asthma may also allow prediction of response to treatment. Researchers from Amsterdam in the Netherlands identified several genetic variants associated with frequent asthma exacerbation despite the use of despite inhaled corticosteroid use (4). If validated by replication this finding will be a useful aid to treatment selection in the near future of widely available clinical GWAS and personalised medicine.

\section{'Environmental' chemicals}

For several decades, researchers have been concerned with the potential adverse respiratory effects of chemicals used in the home. Interest has largely been confined to populations in higher income countries where such chemicals are widely encountered and where competing respiratory risks are relatively few. Two strong examples of such research were presented at this year's ERS meeting. Each focused on a chemical used widely in personal care and cleaning products, in one case limonene (derived from the rind of citrus fruits and a known skin sensitiser), the other 'parabens', a group of antimicrobial compounds used as preservatives. In a survey of 4,000 Canadians (5), of all ages, levels of limonene in household air were associated with wheeze and FeNO, the latter more strongly in children. In Norway (6), measurement of urinary metabolites indicated that exposure to 'parabens' was near ubiquitous and probably far higher in women than men. Regression analysis suggested that levels of exposure influence the oral microbiota in both sexes; and that in 
women this disturbance may be linked to lung function.

While surveys such as these are now increasingly sophisticated, and their findings intriguing, they cannot determine causality which requires other study designs and, usually, the use of toxicology. A nice example of the latter (7) uses two animal models to study the respiratory effects of dibutyl phthalate (DBP), a plasticiser widely used in domestic furnishings and cosmetics. Exposure to an aerosol of DBP induced, through activation of transient receptor potential channels, afferent vagal responses which may be linked directly to cough and bronchospasm.

In useful contrast, researchers in Baltimore, USA, linked symptoms in children with asthma to a 'geocoded' estimate of the distance from their home to the nearest city park (8). On average, children lived a little less than $100 \mathrm{~m}$ from a park; those living closer had fewer 'asthma symptom days', an association which was stronger in older children (6-12 years). The authors suggest that access to green spaceby limiting access, perhaps, to chemicals in the home-may improve asthma control in risk inner-city children.

\section{Acknowledgements}

None.

\section{Footnote}

Conflicts of Interest: The authors have no conflicts of interest to declare.

Cite this article as: Reynolds C, Cullinan P. European Respiratory Society International Congress programme highlights on occupation and epidemiology. J Thorac Dis 2017;9(Suppl 16):S1548-S1549. doi: 10.21037/jtd.2017.11.45

\section{References}

1. Granell R, Henderson J, Sterne J. Genome-wide association study of wheezing phenotypes in the Avon longitudinal study of parents and children. Eur Respir J 2017;50:PA1816.

2. Sayers I, Shrine N, Soler-Artigas M, et al. A Genome Wide Association Study of Moderate-Severe Asthma in subjects from the United Kingdom. Eur Respir J 2017;50:PA1815.

3. Aïssi D, Shao X, Pin I, et al. DNA methylation and lung function: an epigenome-wide association study. Eur Respir J 2017;50:OA2941.

4. Vijverberg SJ, Hernandez-Pacheco N, Farzan N, et al. Genome-wide association study of asthma exacerbations in European children treated with inhaled corticosteroids. Eur Respir J 2017;50:PA1817.

5. Dales R, Cakmak S. Is residential ambient air limonene associated with asthma in the Canadian population? Eur Respir J 2017;50:OA1781.

6. Bertelsen R, Ringel-Kulka T, Peddada S, et al. Oral microbiome and associations with chemical exposure, asthma and lung function. Eur Respir J 2017;50:OA321.

7. Bolaji JA, Bonvini SJ, Wortley MA, et al. Phthalates trigger respiratory reflexes. Eur Respir J 2017;50:PA4785.

8. DePriest K, Butz A, Land C, et al. Access to greenspace and asthma symptoms in urban children with persistent asthma. Eur Respir J 2017;50:PA2647. 